\section{Dünnschicht-chromatographische Trennung und Identifizierung der isomeren Kresole und des Phenols}

Separation and Identification of the Isomeric Cresols and of Phenol by Thin-Layer Chromatography

Trenn. von Kresolen und Phenol; Chromatographie, Dünnschicht

\section{Horst Thielemann}

Eingegangen am 17. Oktober 1972

Über unterschiedliche Möglichkeiten zur dünnschicht-chromatographischen Trennung der Kupplungsprodukte von Phenolverbindungen als Azofarbstoffe konnte bereits berichtet werden [1-4].

Eine besonders schnelle Auftrennung der freien isomeren Kresole und des Phenols gelang an Celluloseschichten, während sich Kieselgel G-, Kieselgel D-, Polyamid- bzw. auch Mischschichten und Fertigfolien UV 254, die sowohl imprägniert als auch aktiviert $(0,5$ und $1 \mathrm{~h})$ waren, nicht oder nur bedingt eigneten. Die 6 isomeren Dimethylphenole (Xylenole), außerdem 1- und 2-Naphthol beeinflussen bei Einhaltung der im experimentellen Teil angegebenen Reaktionsbedingungen die Trennung nicht (Substanzen laufen mit der Laufmittelfront bzw. liegen kurz darunter). Die Polyphenole: 1,2-Dihydroxybenzol (Brenzcatechin), 1,3-Dihydroxybenzol (Resorcin), 5-Methylresorcin (Orcin), 1,4-Dihydroxybenzol (Hydrochinon), 1,2,3-Trihydroxybenzol (Pyrogallol), 1,3,5-Trihydroxybenzol (Phloroglucin) bleiben unter den angegebenen Reaktionsbedingungen am Startpunkt (Ausnahme 1,2-Dihydroxybenzol).

\section{Experimenteller Teil}

Zur Herstellung der Platten empfiehlt es sich, auf Cellulosepulver zur Dünnschicht-Chromatographie (FND - ohne Bindemittel der Spezialpapierfabrik Niederschlag des VEB Freiberger Zellstoff- und Papierfabrik zu Weißenborn) zurückzugreifen.

$25 \mathrm{~g}$ Pulver wurden in $60 \mathrm{ml}$ dest. Wasser (ausreichend für 5 Platten, $20 \times 20 \mathrm{~cm}$ ) $30-60 \mathrm{sec}$ mit einem elektrischen Mixgerät aufgeschlagen und danach sofort auf die Platten in der gewünschten Schichtdicke aufgetragen. Die nassen Schichten können nur an der Luft getrocknet werden.

Für das Sichtbarmachen der aufgetragenen Substanzen eignete sich diazotierte Sulfanilsäure, gelöst in 5\% iger Natriumcarbonatlösung. Die Lösung muß stets frisch hergestellt werden. Bei stärkerer Sodakonzentration tritt eine Braunfärbung der Celluloseschicht ein und verhindert bzw. beeinträchtigt die Identifizierung der Substanzen.
Tabelle 1. $h R_{\text {- }}$-Werte der isomeren Kresole und des Phenols an unterschiedlichen Schichtmaterialien [Laufmittel: Methylenchlorid/Cyclohexan (55:45)]

\begin{tabular}{lccccccc}
\hline $\begin{array}{l}\text { Phenol- } \\
\text { verbindungen }\end{array}$ & A & B & C & D & E & F & G \\
\hline Phenol & 15 & 16 & a & 17 & 70 & b & a \\
o-Kresol & 33 & 32 & a & 31 & 92 & b & a \\
m-Kresol & 30 & 22 & a & 22 & 86 & b & a \\
p-Kresol & 20 & 21 & a & 22 & 81 & b & a \\
& c & c & d & c & d & c & e \\
\hline
\end{tabular}

a Starke Schweifbildung.

b Laufmittelfront, teils Startpunkt.

- Alle aufgetragenen Polyphenole bleiben am Startpunkt.

d 1,2-Dihydroxybenzol (Brenzcatechin) $h_{\mathrm{f}}$-Wert 6 , alle anderen Polyphenole Startpunkt.

e 1,2-Dihydroxybenzol (Brenzcatechin) $\mathrm{hR}_{\mathrm{f}}$-Wert 9, alle anderen Phenole Startpunkt.

$A=$ Kieselgel $\mathrm{G}$ nach Stahl - Schichten imprägniert mit einem Gemisch von Formamid/Aceton $(1: 2)$, Laufzeit $1 \mathrm{~h}$ (o- und m-Kresol ließen sich nicht trennen).

B = Fertigfolien UV 254 der Fa. Sklárny Kavalier (ČSSR). Zusammensetzung: Sorptionsmittel: Kieselgel für Chromatographie mit einem Luminescenzindicator; Unterlage: Aluminiumfolie; Bindemittel: Stärke.

$\mathrm{C}=$ Fertigfolie UV 254 - imprägniert mit Formamid/ Aceton (1:2).

$\mathrm{D}=$ Fertigfolien UV 254 - aktiviert 0,5 bzw. $1 \mathrm{~h}$ (m- und p-Kresol ließen sich nicht trennen).

$\mathrm{E}=$ Celluloseschichten.

$\mathrm{F}=$ Celluloseschichten - aktiviert $30 \mathrm{~min}$ bei $100^{\circ} \mathrm{C} \mathrm{im}$ Trockenschrank.

$\mathrm{G}=$ Celluloseschichten - imprägniert mit Formamid/ Aceton (1:2).

Die $h R_{p}$-Werte für die einzelnen Phenolverbindungen können der Tabelle entnommen werden. Besonders darauf hinzuweisen wäre, daß die Trennung der isomeren Kresole und des Phenols stets mit einem frisch hergestellten Lösungsmittelsystem vorgenommen werden sollte, da sonst beträchtliche Schwankungen hinsichtlich der $h R_{\mathrm{p}}$-Werte und somit der Trennung der Substanzen auftreten können.

\section{Literatur}

1. Thielemann, H.: Z. Chem. 9, 350 (1969).

2. Thielemann, H.: Wiss. Z. Univ. Halle, XVIII'M, 136 (1969).

3. Thielemann, H.: J. Prakt. Chem. 312, 728 (1970).

4. Thielemann, H.: diese Z. 253, 124 (1971).

Dr. rer. nat. H. Thielemann

DDR-36 Halberstadt, Wasserturmstraße 10

Deutsche Demokratische Republik 